\title{
Síntesis y Actividad Antimalárica de Estirilquinolinas sobre Plasmodium falciparum
}

\author{
Gilmar G. Santafé(1)* Emy L. Sánchez ${ }^{(1)}$ y Omar L. Torres ${ }^{(2)}$ \\ (1) Grupo de Investigación "Química de los Productos Naturales”, Departamento de Química, Universidad \\ de Córdoba, Cra. 6 № 74-103, Córdoba-Colombia. (e-mail: gsantafe@correo.unicordoba.edu.co; \\ elsanchez@correo.unicordoba.edu.co) \\ (2) Grupo de Investigación Desarrollo de Fármacos y Afines "IDEFARMA", Departamento de Regencia de \\ Farmacia, Universidad de Córdoba, Cra. 6 № 74-103, Córdoba-Colombia. (e-mail: \\ omart2365@gmail.com)
}

* Autor a quien debe dirigirse la correspondencia

Recibido Ago. 3, 2015; Aceptado Oct. 14, 2015; Versión final Dic. 11, 2015, Publicado Abr. 2016

\section{Resumen}

Con el propósito de encontrar nuevas moléculas activas sobre la malaria, se sintetizaron las estirilquinolinas 2-[(E)-2-(2-metoxifenil)etenil]quinolina (1), 2-[(E)-2-(2-clorofenil)etenil]quinolina (2) y 2[(E)-2-(4-isopropilfenil) etenil] quinolina (3) a partir de la reacción de condensación tipo Perkin entre quinaldina y aldehídos aromáticos. A los compuestos (1), (2) y (3) se les evalúo su actividad antimalárica in vitro frente a las cepas FCB-2 (resistente a la cloroquina) y NF-54 de $P$. falciparum (sensible a la cloroquina) empleando el método radioisotópico. Se encontraron valores de $\mathrm{IC}_{50}$ entre 50,19 y $38,46 \mu \mathrm{M}$ para la cepa FCB-2 y entre 52,49 y 33,70 $\mathrm{MM}$ para NF-54. Las estirilquinolinas mostraron altos porcentajes de inhibición y rendimientos de reacción entre el 60 y 75\%, y las estructuras de cada uno de los productos sintetizados se confirmaron mediante métodos espectroscópicos, principalmente resonancia magnética nuclear. El compuesto (3) mostró la mayor actividad antimalárica con $\mathrm{IC}_{50}=38,46 \mu \mathrm{M}$ para la cepa FCB-2 y 33,70 $\mathrm{MM}$ para NF-54.

Palabras clave: estirilquinolinas; quinaldina; Plasmodium falciparum; actividad antimalárica; reacción de Perkin.

\section{Synthesis and Antimalarial Activity of Styrylquinolines on Plasmodium Falciparum}

\begin{abstract}
With the aim of finding novel active molecules on malaria, three styrylquinolines were synthesized: (1) 2$[(E)$-2-(2-methoxyphenyl)ethenyl]quinolone, (2) 2-[(E)-2-(2-chlorophenyl)ethenyl]quinolone and (3) 2-[(E)2-(4-isopropylphenyl)ethenyl]quinolones. For this, Perkin type condensation reactions between quinaldine and the aromatic aldehydes were done. The antimalarial activity in vitro of the compounds (1), (2) and (3) against strains FCB-2 (resistant to chloroquine) and NF-54 of $P$. falciparum (sensitive to chloroquine) were evaluated by radioisotopic methods. The values $\mathrm{IC}_{50}$ obtained for the strain FCB-2 were between $50.19 \mathrm{y}$ $38.46 \mu \mathrm{M}$; and between for NF-54 were from 52.49 to $33.70 \mu \mathrm{M}$. The styrilquinolines showed high percentages of inhibition and reaction yields between $60 \%$ and $75 \%$, and the structures of the synthesized products were confirmed by spectral methods, mainly nuclear magnetic resonance. The compound (3) showed the highest antimalarial activity with $\mathrm{IC}_{50}=38.46 \mu \mathrm{M}$ for the strain FCB-2 and 33.70 $\mu \mathrm{M}$ for NF-54.
\end{abstract}

Keywords: stirylquinolines; quinaldine; Plasmodium falciparum; antimalarial activity; Perkin reaction 


\section{INTRODUCCIÓN}

En términos de salud y bienestar humano, la malaria sigue siendo una de las más importantes amenazas para el mundo en desarrollo, afectando a millones de personas, cobrando miles de muertes anuales y perjudicando principalmente a naciones subdesarrolladas altamente pobladas y con pocos recursos económicos (López, 2012; González et al., 2012). Esta enfermedad infecciosa es causada por un protozoo intracelular del género Plasmodium, del cual se conocen cinco especies que causan infección en los humanos: Plasmodium falciparum, P. vivax, P. ovale, P. malariae (Celis et al., 2003; Morales et al., 2014) y la más recientemente descubierta $P$. knowlesi, originaria de los primates (Coxet al., 2008, López et al., 2013), siendo $P$. falciparum el más letal. A pesar de haberse presentado una tendencia general de reducción en la incidencia de la infección por malaria, aún las metas propuestas en el plan de acción global contra esta infección liderado por la OMS con base en los planes locales no se han alcanzado, por el contrario, en algunos países y regiones se han observado retrocesos en el proceso (Fernández et al., 2014). Esta tendencia es una consecuencia lógica de que acciones tales como las intervenciones de control tradicionalmente centradas en el diagnóstico y en tratamientos tempranos, la reducción de la transmisión mediante el uso de toldillos tratados con insecticidas y el control del vector, así como en las medidas ambientales y de mejoramiento de las condiciones sanitarias de la población en riesgo, no han logrado el impacto esperado (Piñeros, 2010; Fernández et al., 2014). Por su parte, las políticas públicas de salud aún no abordan el problema de manera integral, con lo cual han contribuido al atraso e incumplimiento de las estrategias con las cuales se pretende erradicar la enfermedad (Molineros et al., 2014).

Actualmente, debido a los problemas de resistencia que presentan los parásitos a algunos de los medicamentos utilizados para su tratamiento (Vicente et al., 2007; Arroyabe et al., 2014), se hace necesario buscar nuevos agentes antimaláricos ya sean de origen natural o sintético, dado que la diversidad y complejidad genética presente en el parasito le confiere a Plasmodium la capacidad de evadir la respuesta inmune del hospedador, produciendo así variantes resistentes a los medicamentos y vacunas, originándose la supervivencia del parásito en la historia evolutiva, así como del fracaso de las estrategias empleadas con el objetivo de erradicarlo (Jiménez et al., 2005), convirtiendo esta resistencia en uno de los mayores retos que enfrenta el control de la malaria (Chugh et al., 2015).

El descubrimiento de la quinina, el primer compuesto que mostró una significativa actividad sobre la malaria, se consolidó como el punto de partida para el desarrollo de nuevos agentes antimaláricos con núcleos quinolínicos y que además exhibieron una importante actividad sobre Plasmodium (Kouznetsov y Amado, 2008). Entre estos derivados quinolínicos están la cloroquina (CQ) el primer análogo sintético de la quinina que inicialmente demostró ser un tratamiento excelente para la malaria (Woster, 2001; Castro y Navarro, 2012), la quinidina, pamaquina, mepraquina, mefloquina y amodiaquina, las cuales se caracterizan estructuralmente por la presencia de un anillo quinolínico y por la actividad sobre las diferentes etapas hepáticas o sanguíneas de Plasmodium (Kouznetsov y Amado, 2008; Torres et al., 2014). Es así como la síntesis de nuevos compuestos se ha convertido en una alternativa para la posible solución de problemas asociados a las limitaciones de tipo farmacológico, como la toxicidad variable y efectos colaterales, aunque el principal problema para los agentes antimaláricos sigue siendo la resistencia por parte del parásito.

En esta búsqueda y desarrollo de nuevos antimaláricos las estirilquinolinas representan una alternativa que busca la obtención de estructuras químicas que puedan aplicarse al desarrollo de antimaláricos potencialmente activos no solo sobre Plasmodium falciparum sino sobre otras especies de protozoos, tomando como punto de referencia las múltiples aplicaciones de la quinolina y sus derivados en el ámbito de las enfermedades protozoarias, en el cual las estirilquinolinas, han sido reportadas por su actividad biológica destacándose las actividades antimalárica, antichagas y leishmanicida (Osorio, 2006; Musiol et al., 2007; Mesa, 2008; ; Sáez et al., 2010; Sánchez et al., 2014), así como también actividades antituberculosis, antiinflamatoria, anticáncer, antibiótica, anti hipertensiva y anti VIH (Zora y Veliog lu, 2008; Swaran et al., 2010). En consecuencia, en esta investigación se obtuvieron tres compuestos tipo estirilquinolinas (2-[(E)-2-

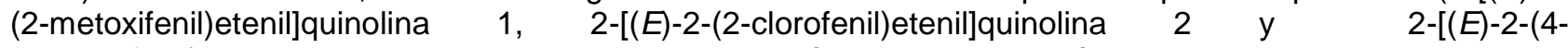
isopropilfenil)etenil]quinolina 3 , empleando una reacción de condensación tipo Perkin entre quinaldina y aldehídos aromáticos, estos productos de síntesis mostraron actividad antimalárica al ser evaluados contra la cepa de $P$. falciparum NF-54 sensible a la cloroquina y la cepa cloroquino resistente FCB-2.

\section{MATERIALES Y MÉTODOS}

\section{Síntesis de estirilquinolinas}

Para la síntesis de 2-[(E)-2-(2-metoxifenil)etenil]quinolina 1, 2-[(E)-2-(2-clorofenil)etenil]quinolina 2 y 2-[(E)2-(4-isopropilfenil)etenil]quinolina 3, obtenidas mediante reacciones de condensación (figura 1), se emplearon como reactivos de partida quinaldina, anhídrido acético, 2-metoxibenzaldehído, 2clorobenzaldehído y 4-isopropilbenzaldehído grado analítico (tipo MERK) respectivamente. 


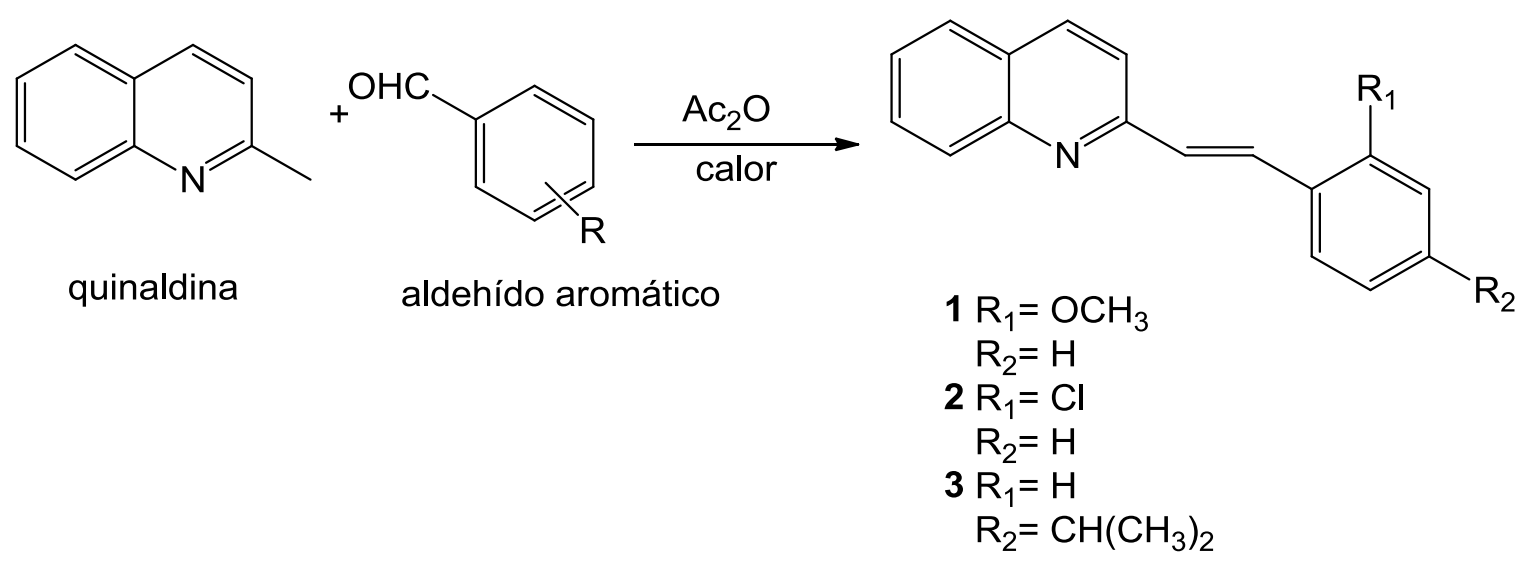

Fig.1: Estructuras de las estirilquinolinas sintetizadas.

A una solución de 7,39mol de quinaldina en $12 \mathrm{ml}$ de anhídrido acético, se le adicionaron 8,3 moles del aldehído correspondiente; la solución se calentó a reflujo por 16 horas y fue monitoreada periódicamente por cromatografía en capa delgada sobre sílica gel (Merck Kiesegel 60 F254), utilizando como revelador el reactivo de Dragendorff. Una vez concluida la reacción, la mezcla se enfrió a temperatura ambiente y se neutralizó con una solución saturada de $\mathrm{NaHCO}_{3}$. Posteriormente el derivado quinolínico se extrajo con $50 \mathrm{ml}$ de la mezcla Hexano:Acetato de etilo en proporción (2:1), con 5 repeticiones. La fase orgánica se secó sobre sulfato de sodio anhidro y finalmente se concentró a sequedad (Benard et al., 2004; Mesa et al., 2008, Sánchez et al., 2014). El producto crudo se purificó por cromatografía en columna (CC), utilizando como eluente mezcla de Bencina de petróleo: Acetato de etilo en proporciones variadas.

La estructura de las moléculas sintetizadas se confirmó mediante técnicas espectroscópicas de Resonancia Magnética Nuclear RMN $-{ }^{13} \mathrm{C}, \mathrm{RMN}-{ }^{1} \mathrm{H}, \mathrm{HMQC}$ y COSY en un espectrómetro Brucker de $400 \mathrm{MHz}$, utilizando como disolvente cloroformo deuterado $\left(\mathrm{CDCl}_{3}\right)$. Los desplazamientos químicos $(\delta)$ se expresaron en partes por millón (ppm) tomando como referencia el TMS y las constantes de acoplamiento (J) en Hercios (Hz).

\section{Preparación de soluciones de las estirilquinolinas 1-3 para evaluación de la actividad antimalárica}

Se preparó una solución madre de $10 \mathrm{mg} / \mathrm{ml}$ de cada una de las estirilquinolinas a evaluar, tomando $2 \mathrm{mg}$ de cada derivado quinolínico y $200 \mu \mathrm{l}$ de dimetil sulfóxido (DMSO) puro. A partir de estase tomaron $50 \mu \mathrm{l}$ y se ajustaron hasta $1000 \mu \mathrm{l}$ con RPMI completo obteniéndose una nueva solución con una concentración final de $500 \mu \mathrm{g} / \mathrm{ml}$, de la cual se prepararon por dilución siete concentraciones para las estirilquinolinas 1-3, en un rango entre $100 \mu \mathrm{g} / \mathrm{ml}$ y $1,56 \mu \mathrm{g} / \mathrm{ml}$. La concentración de DMSO en la primera dilución fue del $1 \%$ y menor en las diluciones sucesivas. Como control de tratamiento se utilizó cloroquina, evaluada en siete diluciones seriadas en un rango entre $1,93 \mu \mathrm{g} / \mathrm{ml}$ y $0,03 \mu \mathrm{g} / \mathrm{ml}$. En este rango de concentraciones existe una relación dosis respuesta y los porcentajes de inhibición del crecimiento están entre el $30 \%$ y el $80 \%$.

\section{Evaluación de la actividad antimalárica in vitro}

La evaluación de la actividad antimalárica in vitro para las estirilquinolinas 1-3 se evalúo sobre las cepas FCB-2 (resistente a la cloroquina) y NF-54 (sensible a la cloroquina) de Plasmodium falciparum por el método radioisotópico, que se fundamenta en la medición indirecta de la actividad metabólica del parásito, por medio de la incorporación de un precursor de ácidos nucleicos marcado radiactivamente. Plasmodium no sintetiza de novo las purinas y por lo tanto debe obtenerlas de una fuente exógena. La ${ }^{3} \mathrm{H}$-Hipoxantina es el precursor de purinas que se emplea en este método para determinar la incorporación en el material genético del parásito y por tanto es un indicador de la replicación del mismo (Trager y Jensen, 1976; Desjardinset al., 1979; Deharo et al., 2000;Mesa et al., 2011).

La evaluación de la actividad antimalárica se realizó en placas de 96 pozos, para ello se preparó una suspensión de glóbulos rojos parasitados con un hematocrito del $2 \%$ y una parasitemía del $1 \%$. La concentración final de la hipoxantina tritiada por pozo fue del $2 \mu \mathrm{Ci} / \mathrm{ml}$. El cultivo con los tratamientos se incubó a $37^{\circ} \mathrm{C}$ durante 48 horas en un atmósfera al $5 \%$ de $\mathrm{CO}_{2}, 5 \%$ de $\mathrm{O}_{2}$ y nitrógeno balanceado, el sistema se congeló a $-20^{\circ} \mathrm{C}$ con el objetivo de provocar la hemólisis de los eritrocitos (García, 2013). Inicialmente se colocaron $100 \mu \mathrm{L}$ de la solución madre de cada compuesto en la fila $\mathrm{H}$ y se hicieron diluciones seriadas en los pozos G-B, con $50 \mu \mathrm{L} /$ pozo. La fila $A$ se toma como control negativo o de crecimiento $(\mathrm{CN})$, para este se preparó una suspensión de glóbulos rojos parasitados con un hematocrito del $2 \%$ y una parasitemia del $1 \%$. Todas las evaluaciones (tratamientos, control y cloroquina) se evaluaron 
por duplicado en cada plato y se realizaron tres ensayos independientes. Haciendo uso de un colector "cell harvesters" se colectaron los ácidos nucleicos y fueron depositados en un filtro de fibra de vidrio y posteriormente las cuentas por minuto $(\mathrm{cpm})$ fueron leídas en un plate chameleon microplate reader Hidex®.

El resultado de actividad antimalárica se determinó con base en el cálculo del porcentaje de inhibición (\%l) de la parasitemia para cada concentración de estirilquinolina; en el cuál se compara el crecimiento de la parasitemia en presencia del derivado quinolínico con la parasitemia del control de crecimiento (control negativo). El porcentaje de inhibición se calculó utilizando la ecuación 1:

$\%$ Inhibición= $100-[(\mathrm{P} / \mathrm{T}) \times 100]$

En donde $\mathrm{P}$, corresponde al promedio de cpm en duplicado (cuentas por minuto) e indica que tan metabólicamente activo son a cada concentración y $\mathrm{T}$ : es el control Negativo (cultivo de glóbulos parasitados sin droga).

El cálculo de la Concentración mínima inhibitoria $\left(\mathrm{IC}_{50}\right)$ se determinó a través de una interpolación lineal a partir de la ecuación 2.

$\log (\mathrm{IC} 50)=\log (X 1)+\frac{50-Y 1}{Y 2-Y 1}(\log X 2-\log X 1)$

Donde, $\mathrm{X} 1$ corresponde a la concentración del compuesto que da una inhibición de la parasitemia Y1 > 50\%; X2 corresponde a la concentración del compuesto que da una inhibición de la parasitemia Y2 <50\%.

\section{RESULTADOS Y DISCUSIÓN}

Las estirilquinolinas $1-3$, se obtuvieron con rendimientos de $75,4 \%, 74,3 \%$ y $60 \%$ respectivamente. La síntesis se llevó a cabo siguiendo la metodología descrita. Todos los compuestos sintetizados mostraron un rendimiento por encima del $50 \%$, esta tendencia se manifiesta en las estirilquinolinas formadas a partir de quinaldinas, a diferencia de los rendimientos reportados para algunas moléculas de este tipo obtenidas a partir de 8-hidroxiquinaldina, para las cuales se reportan rendimientos mucho más bajos (Torres et al., 2014), por lo tanto, el rendimiento de la reacción está directamente relacionado con la presencia o ausencia en la posición 8 del heterociclo inicial del grupo hidroxi, en el cual sus electrones libres interaccionan con el sistema $\pi$ del anillo dispersando una carga negativa en la molécula haciéndola menos reactiva a la acción de sustancias básicas. Este efecto no se refleja en ausencia del hidroxi, por lo tanto para el caso de la quinaldina, los protones del metilo ubicado en la posición dos pueden reaccionar más fácilmente con la base utilizada permitiendo el incremento en el rendimiento de reacción de las correspondientes estirilquinolinas. La estructura de las moléculas sintetizadas confirmada mediante experimentos de resonancia magnética nuclear en una y dos dimensiones, se relaciona a continuación de acuerdo a la numeración descrita en la figura 2.

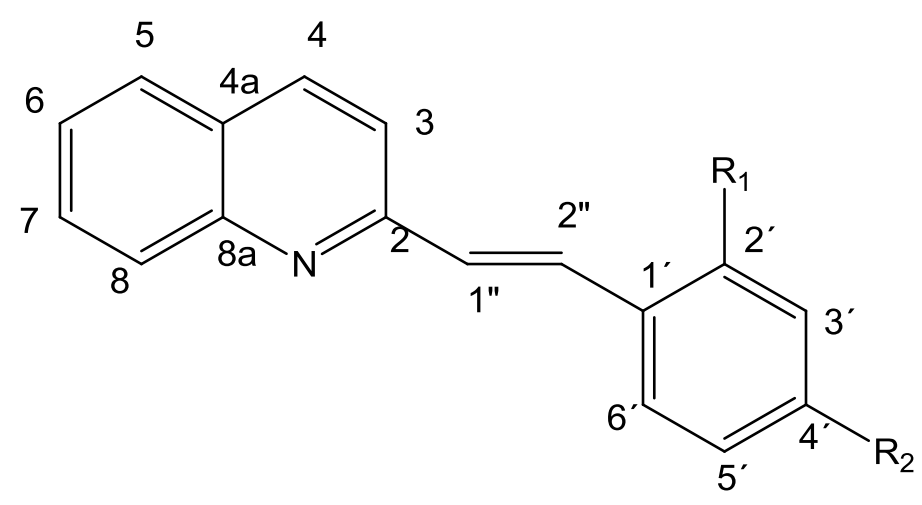

Fig.2: Numeraciónde estirilquinolinas

\section{Datos espectroscópicos de las estirilquinolinas sintetizadas}

2-[(E)-2-(2-metoxifenil)etenil]quinolina 1. Cristales blancos, $\mathrm{C}_{18} \mathrm{H}_{15} \mathrm{NO} .261$ u.m.a. RMN ${ }^{1} \mathrm{H} \delta(\mathrm{ppm}) \delta 3.91$ (s, $3 \mathrm{H}, \mathrm{OCH}_{3}$ ); 6.92 (d, J=8.26 Hz, 1H, H6'); 7.01 (t, J=7.5 Hz, 1H, H4'); 7.47 (d, J= $\left.16.58 \mathrm{~Hz}, 1 \mathrm{H}, 2^{\prime \prime}\right) ; 7.48$ (t, J=7.61 Hz, 1H, H6); 7.31 (t, J=7.5 Hz, 1H, H5'), $7.71-7.76(\mathrm{~m}, 3 \mathrm{H}, \mathrm{H} 3, \mathrm{H} 5$ y H3'); 7.68 (t, J=7.39 Hz, 1H, $\mathrm{H} 7) ; 8,03$ (d, J=16.58 Hz, 1H, H1"); 8.10 (d, 2H, H4 y H8). RMN ${ }^{13} \mathrm{C} \delta$ (ppm) $\delta 157.4$ (C2); 127.1 (C3); 129.1 
(C4); 127.4 (C4a); 125.5 (C5); 125.9 (C6); 129.7 (C7); 136.1 (C8); 148.2 (C8a); 125.4 (C1'); 156.7 (C2'); 118.9 (C3'); 120.7 (C4'); 127.2 (C5'); 110.9 (C6'); 129.3 (C1"); 129.5 (C2"); $55.4\left(\mathrm{CH}_{3}\right)$.

2-[(E)-2-(2-clorofenil)etenil]quinolina 2. Cristales amarillos, $\mathrm{C}_{17} \mathrm{H}_{12} \mathrm{NCl}$. 265.45 u.m.a. RMN ${ }^{1} \mathrm{H} \delta(\mathrm{ppm}) \delta$ $7.45(\mathrm{~d}, \mathrm{~J}=8.75 \mathrm{~Hz}, 1 \mathrm{H}, \mathrm{H} 3) ; 8.13(\mathrm{~d}, \mathrm{~J}=8.50 \mathrm{HZ}, 1 \mathrm{H}, \mathrm{H} 4) ; 7.86-7.75$ (m, 2H, H3'y H5); 7.51 (t, J= 7.87 Hz, $1 \mathrm{H}, \mathrm{H6}) ; 7.73(\mathrm{t}, \mathrm{J}=8.19 \mathrm{~Hz}, 1 \mathrm{H}, \mathrm{H} 7) ; 8.16(\mathrm{~d}, \mathrm{~J}=8.64 \mathrm{~Hz}, 1 \mathrm{H}, \mathrm{H} 8) ; 7.32$ (t, J=7.56 Hz, 1H, H4'); 7.84 (t, J= $\left.7.71 \mathrm{~Hz}, 1 \mathrm{H}, \mathrm{H} 5^{\prime}\right) ; 7.28$ (d, J=7.31 Hz, 1H, H6'); 8.07 (d, J= $\left.16.39 \mathrm{~Hz}, 1 \mathrm{H}, \mathrm{H} 1^{\prime \prime}\right) ; 7.45$ (d, J= 16.25 Hz, $1 \mathrm{H}$, H2"). RMN ${ }^{13} \mathrm{C} \delta(\mathrm{ppm}) 155.7$ (C2); 129.8 (C3); 136.4 (C4); 127.5 (C4a); 118.9 (C5); 126.4 (C6); 129.9 (C7); 129.2 (C8); 148.1 (C8a); 134.0 (C1'); 134.5 (C2'); 127.4 (C3'); 127.0 (C4'); 126.9 (C5'); 129.4 (C6'); 130.2 (C1"); 131.6 (C2").

2-[(E)-2-(4-isopropilfenil)etenil]quinolina 3. Cristales amarrillos, $\mathrm{C}_{20} \mathrm{H}_{19} \mathrm{~N} .273$ u.m.a. $\mathrm{RMN}{ }^{1} \mathrm{H} \delta(\mathrm{ppm}) \delta$ $7.34(\mathrm{~d}, \mathrm{~J}=8,20 \mathrm{~Hz}, 1 \mathrm{H}, \mathrm{H3}) ; 8.13(\mathrm{dd}, \mathrm{J}=8.696 \mathrm{~Hz}, 2 \mathrm{H}, \mathrm{H} 4$ y H8); 7.79 (d, J= $7.98 \mathrm{HZ}, 1 \mathrm{H}, \mathrm{H} 5) ; 7.52$ (t, J= $7.61 \mathrm{~Hz}, 1 \mathrm{H}, \mathrm{H6}) ; 7.74(\mathrm{~m}, 1 \mathrm{H}, \mathrm{H} 7) ; 7.58$ (d, J=8.09 Hz, 2H, H2'y H6'); 7.27 (d, J=8.16 Hz, 2H, H3'y H5'); 7.68 (d, J=16.3 HZ, 1H, H1"); 7.42 (d, J = $16.36 \mathrm{~Hz}, 1 \mathrm{H}, \mathrm{H} 2$ "); 2.94 (sept, 1H, CH isopropil); 1.28 (d, $6 \mathrm{H}$, $\mathrm{CH}_{3}$ ). $\quad \operatorname{RMN}{ }^{13} \mathrm{C} \delta(\mathrm{ppm}) 156.2$ (C2); 126.4 (C3); 128.9 (C4); 129.7 (C4a); 127.4 (C5); 126.0 (C6); 119.0 (C7); 136.3 (C8); 149.7 (C8a); 134.1 (C1'); 127.3 (C2'); 126.8 (C3'); 148.1 (C4'); 126.8 (C5'); 127.3 (C6'); 134.5 (C1");127.9 (C2"); 34.2 (CH grupo isopropilo); $24.1\left(2 \times \mathrm{CH}_{3}\right)$

\section{Evaluación de la actividad antimalárica}

Los resultados de la evaluación de la actividad antimalárica de las estirilquinolinas sintetizadas se reportan en la tabla 1.

Tabla 1: Resultados de la evaluación de la actividad antimalárica sobre las cepas FCB-2 y NF-54 de Plasmodium falciparum ${ }^{*} \mathrm{CQ}$ cloroquina en unidades $\mathrm{nM}$

\begin{tabular}{|c|c|c|c|c|c|c|}
\hline \multirow{2}{*}{ Estirilquinolinas } & \multicolumn{3}{|c|}{ Cepa FCB - } & \multicolumn{3}{c|}{ Cepa NF - 54 } \\
\cline { 2 - 7 } & $\begin{array}{c}\mathrm{IC}_{50} \\
(\mu \mathrm{g} / \mathrm{ml})\end{array}$ & $\mathrm{IC}_{50}(\mu \mathrm{M})$ & $\% \mathrm{I}$ & $\mathrm{IC}_{50}(\mu \mathrm{g} / \mathrm{ml})$ & $\mathrm{IC}_{50}(\mu \mathrm{M})$ & $\% \mathrm{I}$ \\
\hline 1 & 13,10 & 50,19 & 91,70 & 13,70 & 52,49 & 90,50 \\
\hline 2 & 11,60 & 43,77 & 92 & 12,10 & 45,66 & 89,30 \\
\hline 3 & 10,50 & 38,46 & 91,20 & 9,20 & 33,70 & 88,30 \\
\hline $\mathrm{CQ}$ & $* 102,2$ & 0,102 & 86,40 & ${ }^{*} 7,8 \times 10^{-12}$ & $7,8 \times 10^{-15}$ & 86 \\
\hline
\end{tabular}

Las estirilquinolinas 1-3 fueron evaluadas sobre las cepas FCB - 2 y NF - 54, evidenciándose a partir de los valores de $\mathrm{IC}_{50}$ calculados (Tabla 1 ) que a altas concentraciones de estas moléculas se inicia una reducción significativa de la parasitemia lo que se refleja en una baja actividad contra Plasmodium, sin embargo a pesar de tener valores altos de $\mathrm{IC}_{50}$, los compuestos son activos contra el parásito, debido a que muestran porcentajes máximos de inhibición por encima del 80\% (Tabla 1), además presentan una buena relación dosis-respuesta para cada una de la cepas evaluadas. De acuerdo con los resultados obtenidos, las variaciones en los sustituyentes sobre el anillo inicialmente presentes en los aldehídos empleados, aunque influyen en la actividad de la molécula sobre el parásito, en este caso no originan una disminución apreciable en los valores de $\mathrm{IC}_{50}$ calculados; destacándose que la mayor actividad de las moléculas sobre las cepas FCB-2 y NF - 54 la presenta la estirilquinolina 3, la cual tiene un grupo isopropil como sustituyente en el anillo aromático; y valores $I_{50}$ de $38,46 \mu \mathrm{M}$ y $33,70 \mu \mathrm{M}$ respectivamente. Los valores sugieren que la sustitución en la posición 2 del anillo puede estar a la vez asociada con la leve disminución de la actividad sobre el parásito, resaltando que las estirilquinolinas 1 y 2 son igualmente activas sobre las cepas evaluadas.

Previamente, Torres et al (2014) reportaron valores de $\mathrm{IC}_{50}$ de 15,4 $\mu \mathrm{M}$ para la estirilquinolina 2-[(E)-2-(4isopropilfenil)etenil]quinolin-8-il-acetato, la cual fue evaluada sobre la cepa FCB-2 y cuya diferencia con el compuesto 3 radica en la sustitución sobre el carbono 8 del sistema quinolínico con un $\mathrm{IC}_{50}$ de $38,46 \mu \mathrm{M}$, lo que sugiere que esta sustitución por el grupo acetilo ocasiona un aumento en la actividad antimalárica de estirilquinolinas sobre la cepa evaluada; mientras que sustituyentes tales como el grupo octiloxi, originan una disminución sustancial de la actividad sobre el parásito, así se evidencia en los valores reportados para la estirilquinolina 2-[(E)-2-(4-isopropilfenil)etenil]-8-(octiloxi) quinolina que presenta un valor de $\mathrm{IC}_{50}$ de $144,1 \mu \mathrm{M}$, el cual está muy por encima del calculado para el compuesto 3 (Torres et al.,2014). De otra parte, los valores de $\mathrm{IC}_{50}$ para este mismo compuesto $(38,46 \mu \mathrm{M})$, están muy cercanos a los reportados para el compuesto diosgenona que presentó valores de $\mathrm{IC} 50=27,9 \mu \mathrm{M}$ y $35,4 \mu \mathrm{M}$ en la cepa de Plasmodium falciparum FCB2 y NF54 resistente y sensible a cloroquina, respectivamente (Arroyabe et al., 2014). Es relevante mencionar que productos naturales como los alcaloides Galipinina, Agustureine, Galipeine, 
Cuspareine aislados de Galipea officinalis en cuyas estructuras, al igual que en los compuestos sintetizados en este trabajo, está presente el sistema quinolínico, también mostraron una significativa actividad sobre Plasmodium (Jacquemond-Collet, 2002; Osorio, 2006).

Para establecer el comportamiento metabólico del parásito, se estudió la relación dosis-respuesta de los compuestos 1,2 y 3 , encontrándose que de manera similar a la Cloroquina, a medida que se aumenta progresivamente la concentración de cada uno de ellos se logra inhibir el crecimiento del parásito (figura 3), por lo tanto las estirilquinolinas son compuestos de acción rápida y presentan una buena relación dosisrespuesta.

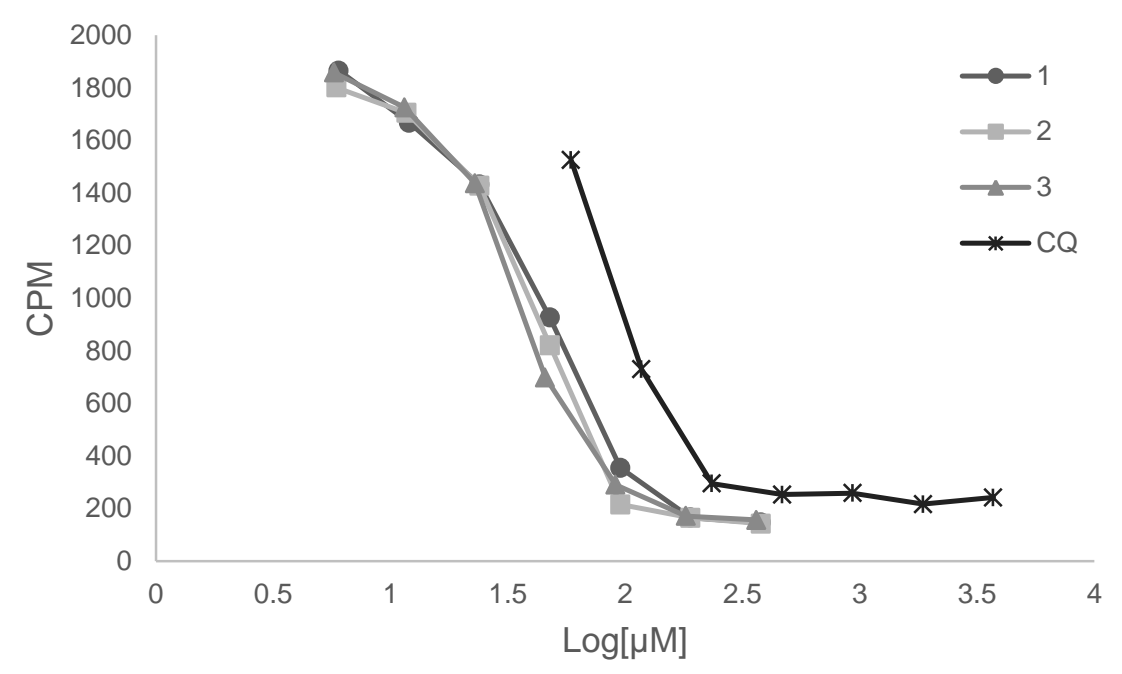

Fig. 3: Curvas de relación dosis Vs respuesta para las estirilquinolina 1,2 y 3 sobre lacepa FCB-2

El comportamiento de cada una de las estirilquinolinas sobre la cepa NF-54 se muestra en la figura 4, evidenciándose también en este caso, una buena relación dosis- respuesta debido a que se observa un aumento progresivo en el porcentaje de inhibición de la parasitemia al aumentar la concentración de los productos evaluados sobre la cepa de Plasmodium.

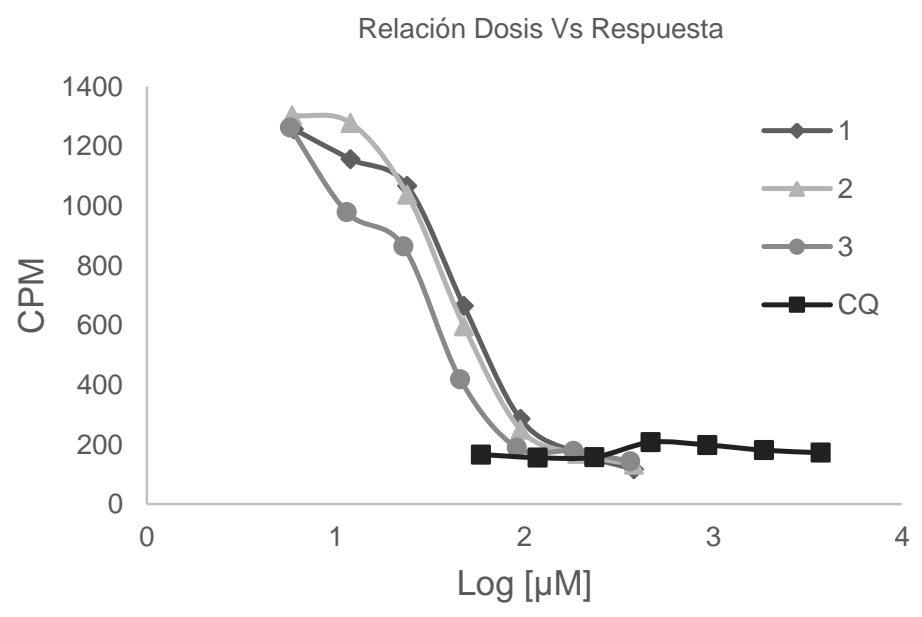

Fig. 4: Curvas de relación dosis Vs respuesta para las estirilquinolina 1,2 y 3 sobre lacepa NF-54

Las estirilquinolinas evaluadas presentan buena actividad sobre las cepas de Plasmodium ensayadas, resaltando que la molécula 2-[(E)-2-(2-clorofenil)etenil]quinolina (2), mostró porcentajes de inhibición de 92 y $89,3 \%$ en las cepas FCB-2 y NF-54 respectivamente a la máxima concentración evaluada, aunque sus IC $_{50}$ corresponden a $43,77 \mu \mathrm{M}$ y $45,66 \mu \mathrm{M}$. Esta actividad puede estar inducida entre otros factores por la presencia del átomo de cloro en la posición dos del anillo procedente del aldehído aromático.

Los resultados alcanzados en este trabajo evidencian y confirman la importancia de evaluar la actividad antimalárica en esta clase de compuestos, ratificándose que esta actividad está basada en la sustitución en la posición 2 del sistema quinolínico, factor que induce la actividad antimalárica, al mismo tiempo se 
evidencia que variaciones en el tipo de sustituyente y sus respectivas posiciones en el anillo aromático aldehídico muestran cambios significativos en los valores de la actividad evaluada (Gantier et al., 1996; Fakhfakh et al., 2003; Martínez et al., 2005; Soto et al., 2011)

\section{CONCLUSIONES}

La obtención de estirilquinolinas mediante la reacción de condensación entre la quinaldina con aldehídos aromáticos en presencia de anhídrido acético, constituye una ruta adecuada para la síntesis de este tipo de compuestos llegando a obtener rendimientos hasta del $75,40 \%$, ratificándose por tanto la importancia y viabilidad de esta ruta sintética.

Los valores de los porcentajes de inhibición reportados para las tres estirilquinolinas evaluadas sobre la cepa cloroquino-resistente (FCB-2) y la cepa sensible a la cloroquina (NF-54), evidenciaron que estas moléculas tienen la capacidad de disminuir notablemente la acción metabólica del parásito, originando con ello una reducción en la parasitemia; este comportamiento deriva directamente de las estructuras de cada uno de estos productos de síntesis ensayados, cuyas variaciones en los sustituyentes sobre el anillo aromático traen consigo cambios significativos en la actividad de la molécula sobre el parásito. Todas las estirilquinolinas evaluadas mostraron potencialidad sobre las cepas de Plasmodium falciparum evaluadas, sin embargo, el compuesto 2-[(E)-2-(4-isopropilfenil)etenil]quinolina, 3 presentó los mayores porcentajes de inhibición, correspondientes a 91,20 y 88,30\% para las cepas FCB-2 y NF-54 respectivamente.

\section{AGRADECIMIENTOS}

A la Universidad de Córdoba, Montería, Colombia, por la financiación de esta investigación.

\section{REFERENCIAS}

Arroyabe, J., S, Blair y N. Restrepo. Hemisíntesis de un híbrido diosgenonaartesunato y su efecto antiplasmodial, VITAE, 21(3), $211-217$ (2014)

Benard, C., y otros once autores, Linker-modified quinoline derivatives targeting HIV-1 integrase: synthesis and biological activity, Bioorganic \& Medicinal Chemistry Letters, 14 (10), 2473 - 2476 (2004)

Castro, W. y M. Navarro, Complejos metálicos como potenciales antimaláricos con blanco de acción: hemozoína, Avances en Química, 7(2), 135 - 144 (2012)

Celis, J. R., R. Montenegro, A. Castillo, E. Che y A. Muños. Evolución de la malaria en la región Loreto, Anales de la Facultad de Medicina, 64(4), 261 - 266 (2003)

Chugh, M., y otros 17 autores, Identification and deconvolution of cross-resistance signals from antimalarial compounds using multidrug-resistant Plasmodium falciparum strains, Antimicrobial Agents and Chemotherapy, 59(2), 1110-1118 (2015)

Cox, J., y otros ocho autores, Plasmodium knowlesi malaria in humans is widely distributed and potentially life threatening. Clinical infectious diseases, 46(2), 165-171 (2008)

Deharo, E., PH. Gautre, V. Muñoz y M. Sauvain, Técnicas de laboratorio para la selección de sustancias antimaláricas, 51 - 52. CYTED-IRD, La Paz, Bolivia (2000)

Desjardins, R., C. Canfield, J. Haynes y J. Chulay, Quantitative assessment of antimalarial activity in vitro by a semiautomated microdilution technique, Antimicrobial Agents and Chemotherapy, 16 (6), 710-718 (1979)

Fakhfakh, M. A. y otros seis autores, Synthesis and Biological Evaluation of Substituted Quinolines: Potencial Treatment of Protozoal and Retroviral Co-infections, Bioorganic \& Medicinal Chemistry, 11(23), 5013-5023 (2003)

Fernández, J., A. Idrovo, V. Giraldo y H. Molina, Aproximación a los saberes no institucionales, Biomédica, 34, 250-259 (2014)

Gantier, J.C., A. Fournet, MH. Muñoz y R. Hocquemiller, The Effect of Some 2-Substituted Quinolines Isolated from Galipea longiflora on Plasmodium vinckei petteri Infected Mice, Planta Medica, 62(3), 285-286 (1996)

García, P., A. Pabón, S. Blair y C. Arias, Evaluación del efecto citotóxico y del daño genético de los extractos estandarizados de Solanum nudum con actividad anti-plasmodium, Biomédica, 33, 78-87 (2013)

González, J., y otros seis autores, La investigación científica en el campo de la malaria, importancia socioeconómica y ética, Panorama Cuba y Salud, 7(3), 28 - 36 (2012) 
Jacquemond, I., y otros 5 autores, Antiplasmodial and Cytotoxic Activity of Galipinine and Other Tetrahydroquinolines From Galipea officinalis, Planta Med. 68 (1), 68-69 (2002)

Jiménez J., C. Muskus y I. Vélez, Diversidad genética de Plasmodium falciparum y sus implicaciones en la epidemiología de la Malaria, Biomédica, 25(4), 588-602 (2005)

Kouznetsov, V. y D. Amado, Antimaláricos: construcción de híbridos moleculares de la Cloroquina, UNIVERSITAS SCIENTIARUM, 13(3), 306 - 320 (2008)

López, M. C. A, Diversity-Oriented Synthesis Yields a Novel Lead for the Treatment of Malaria, Anales de la Real Academia Nacional de Farmacia, 78(4), 418- 424 (2012)

López M., V. Fumadó y M. González, Actualización en el diagnóstico y tratamiento de la malaria, Anales de Pediatría, 78(2), 124.e1-124.e8 (2013)

Martínez, M., y otros siete autores, Nematocidal and trichomonacidal activities of 2-substituted quinolones, IL Farmaco, 60 (3), 219-224 (2005)

Mesa, A., A. Pabón y S. Blair, Actividad antiplasmodial in vitro de Calophyllum inophyllum, Revista QuímicaViva, 2 (10), 118-128 (2011)

Mesa, A., y otros seis autores, Síntesis y Actividades Leishmanicida y Citotóxica in vitro de Análogos 2-

Arilquinolinas, VITAE, 15 (2), 259-266 (2008)

Molineros, L., O. Calvache, H. Bolaños, C. Castillo y C. Torres, Aplicaciones de un modelo integral para el estudio de la malaria urbana en San Andrés de Tumaco, Colombia, Revista Cubana de Medicina Tropical, 66(1), 3-19 (2014)

Morales, M., M. Pérez, H. Guerra, J. Martínez y A. Regalado, Paludismo por Plasmodium vivax: presentación de un caso, Rev. Ciencias Médicas, 18(4), 714-720 (2014)

Musiol, R., y otros nueve autores, Investigating biological activity spectrum for novel quinoline Analogues, Bioorganic \& Medicinal Chemistry, 15 (3), 1280-1288 (2007)

Osorio, E., G. Montoya y G. Arango, Productos Naturales alcaloidales con actividad antiprotozoaria, VITAE, 13 (1), 61-84 (2006)

Piñeros J., Malaria y determinantes sociales de la salud: un nuevo marco heurístico desde la medicina social latinoamericana, Biomédica, 30, 178-87 (2010)

Sáez, J., García, P., Genes, C., Molano, P., Torres, O. y O. Triana, Evaluation of the Trypanocidal, Cytotoxic And Genotoxic Activity of Styrylquinoline Analogs, Journal of Chemotherapy, 22(3), 169-174 (2010)

Sánchez, E., G. Santafé, O. Torres, D. Muños y S. Robledo, Compuestos sintéticos tipo estirilquinolinas con actividad leishmanicida y citotóxica, Biomédica, 34, 605-611 (2014)

Soto, A., J. Meneses, J. Sáez y M. Camargo, Evaluación de citotoxicidad de nuevos análogos de estirilquinolinas en células leucemooides Jurkat, Actualidades Biológicas, 33 (95), 165 - 172 (2011)

Swaran, S., A. Vasudeva, I. Chowdhury, N. Pal y KD. Thomas, New quinoline derivatives: Synthesis and investigation of antibacterial and antituberculosis properties, European Journal of Medicinal Chemistry, 45, 3374-3383 (2010)

Torres, O., R. Espinosa, A. Sáez y G. Santafé, Evaluación de la actividad antimalárica y citotóxica de estirilquinolinas obtenidas a partir de 8-hidroxiquinaldina con aldehídos aromáticos, Información Tecnológica, 25 (6), 91-100 (2014)

Trager, W. y J. Jensen, Human Malaria Parasites in Continuos culture, Science, 193 (4254), 674-675 (1976)

Vicente, E., y otros diez autores, Synthesis and structure activity relationship of 3-phenylquinoxaline 1,4-di$\mathrm{N}$-oxide derivatives as antimalarial agents, European Journal of Medicinal Chemistry, 43, $1903-1910$ (2007)

Woster, P., Annual reports in medicinal chemistry: New therapies for parasitic infection. Academic press, cap. 10, San Diego, 2001

Zora, M. y O. Veliog $ॅ$ lu, Synthesis of ferrocenyl quinolones, Journal of Organometallic Chemistry, 693, 2159$2162(2008)$ 